\title{
La valoración de los servicios ecosistémicos en los ecosistemas forestales: un caso de estudio en Los Alpes Italianos
}

\author{
Enhancement of forest ecosystem services: a case study in the Italian Alps
}

\section{Laura Rodríguez García a, Giorgio Curetti b, Giulia Garegnani b, Gianluca Grilli b,c, Fabio Pastorella ${ }^{\mathrm{d}}$, Alessandro Paletto ${ }^{\text {a* }}$}

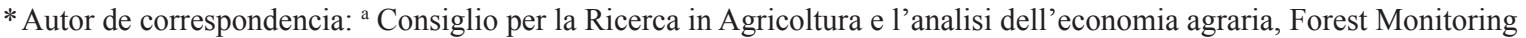
and Planning Research Unit (CREA-MPF), Trento, Italy, phone: +390461381115, alessandro.paletto@entecra.it

${ }^{\mathrm{b}}$ Institute for Renewable Energy, European Academy of Bolzano (EURAC), Bolzano, Italy.

${ }^{c}$ University of Trento, Department of Civil, Environmental and Mechanical Engineering, Trento, Italy.

dEuropean Forest Institute (EFI) Project Center "MOUNTFOR”, San Michele A/A, Italy.
}

\begin{abstract}
SUMMARY
The values of goods and services by natural resources are not included in the political decision making process concerning natural resources management. This gap is due to the fact that many ecosystem services are not marketed. In order to overcome this gap in the management of natural resources, it is necessary to apply some environmental economic methods to capture the total economic value of marketed and non-marketed ecosystem services. Ecosystem services are not homogeneous across landscapes but they are heterogeneous in space. Consequently, mapping ecosystem services is considered as a fundamental requirement for landscape planning. The aim of this paper is to show a method for the economic valuation and mapping of the forest ecosystem services. The method was applied to a case study characterized by a high importance of ecosystem services and involved in the recharge.green project (Alpine Space Programme): the Gesso-Vermenagna valley in Italy. Results show that the highest economic values are for regulating services (from $11 € \mathrm{ha}^{-1}$ to $4.300 € \mathrm{ha}^{-1}$ per year) and for provisioning services (from $6 € \mathrm{ha}^{-1}$ to $1.980 € \mathrm{ha}^{-1}$ per year), while the lowest values are for cultural services (from $6 € \mathrm{ha}^{-1}$ to $627 € \mathrm{ha}^{-1}$ per year). This study can provide useful information to decision makers in order to improve the management of natural resources at local level.
\end{abstract}

Key words: provisioning services, regulating services, cultural services, mapping, economic valuation.

\section{RESUMEN}

El valor de los bienes y servicios suministrados por ecosistemas naturales no se incluye, a menudo, en el proceso político de toma de decisiones sobre manejo de recursos naturales. Esto, debido a que muchos beneficios no se llegan a comercializar. Para evitar este límite en la gestión de los recursos naturales, es necesario desarrollar técnicas que evalúen los servicios ecosistémicos y que capturen el valor económico total de los servicios comerciales y no comerciales. Los servicios ecosistémicos no son heterogéneos a través de los paisajes. Por consiguiente, el conocimiento de la distribución espacial y la importancia económica de los servicios ecosistémicos es una información fundamental para mejorar la planificación de las intervenciones. En el artículo se muestra un procedimiento para la evaluación económica y localización espacial de servicios ecosistémicos que son suministrados por un ecosistema forestal. El método propuesto fue analizado mediante un caso de estudio en el valle del Gesso-Vermagnana, Italia. Este se caracteriza por su importancia en el ámbito de los recursos naturales y por estar involucrado en el proyecto recharge.green (Programa Espacial Alpino). Los resultados muestran que los valores más altos corresponden a los servicios de regulación (11 a $4.300 €$ ha $^{-1}$ año ${ }^{-1}$ ) y a los servicios

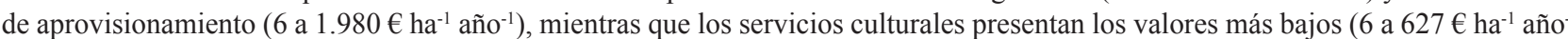
$\left.{ }^{1}\right)$. Este estudio permite ampliar el conocimiento y la información útil para los responsables encargados de la toma de decisiones sobre el manejo de recursos naturales.

Palabras clave: servicios de aprovisionamiento, servicios de regulación, servicios culturales, evaluación espacial, valoración económica.

\section{INTRODUCCIÓN}

Hoy se sabe que la biodiversidad forestal sustenta una amplia gama de bienes y servicios necesarios para el bienestar humano (MEA 2005) como recursos naturales (comida, agua, madera para construcción y bio-energía, piensos y plantas medicinales), polinización, abastecimiento y purificación del agua potable, protección contra los riesgos naturales (deslizamientos de tierra, inundaciones, desprendimientos de rocas y avalanchas), captura y almacenamiento de carbono, y producen una amplia gama de bienes que tienen fines medicinales, culturales y espiri- 
tuales (Fisher et al. 2009). El concepto de servicios ecosistémicos empezó a conocerse a principios de los años 80 , y posteriormente durante los años 90 este concepto ha sido introducido en el debate científico debido a los múltiples autores que lo aplicaban a través de diferentes perspectivas de análisis. Con la Evaluación de los Ecosistemas del Milenio (MEA) en 2005 el concepto consiguió un gran avance más allá del discurso científico. En este artículo, los servicios ecosistémicos son considerados como los procesos y condiciones a través de los cuales los ecosistemas naturales sostienen y satisfacen la vida humana, y los beneficios de las poblaciones humanas provienen, directa o indirectamente, de las funciones que ofrecen los ecosistemas (Costanza et al. 1997). MEA (2005) clasificó los servicios ecosistémicos en base a sus funciones, y se establecieron en cuatro categorías: (1) servicios de aprovisionamiento (o servicios de provisión), en referencia a los productos que pueden ser cosechados como comida, madera, forraje, provisión de agua; (2) servicios de regulación, considerando el papel que desempeñan los ecosistemas en la regulación de los procesos ecológicos (por ejemplo. regulación del agua y el clima); (3) servicios culturales, los cuales suponen los beneficios no materiales proporcionados por los ecosistemas (por ejemplo, actividades recreacionales, valores espirituales y culturales); y por último (4) servicios de apoyo tales como la producción vegetal y el ciclo de nutrientes. Sucesivamente, De Groot et al. (2010) reclasificaron los servicios ecosistémicos reemplazando los servicios de apoyo por servicios de hábitat (por ejemplo, hábitat de crianza, protección del patrimonio genético), con el fin de evitar la doble contabilidad de un mismo servicio (Hein et al. 2006). Las decisiones relacionadas con la gestión o el uso de la tierra deberían estar basadas en dos aspectos fundamentales, teniendo en especial consideración las diferentes categorías de los servicios ecosistémicos (Eade y Moran 1996): (i) el valor comerciable y no-comerciable de los bienes y servicios de los ecosistemas, (ii) y la distribución espacial de estos valores.

El valor de los bienes y servicios frecuentemente está poco considerado en la toma de decisiones sobre el manejo de recursos naturales, debido a la falta de conocimiento en los valores que están atribuidos al bienestar humano (GrêtRegamey et al. 2008). Analizar las propiedades de los ecosistemas, derivadas respectivamente de los servicios del ecosistema y de su valor de mercado, puede ayudar a informar a las instituciones para la última fase decisional.

Sin embargo, existen problemas para definir su valor exacto, por ejemplo, en la comparación de los costos de explotación y de conservación de los recursos naturales a largo plazo. Con el fin de superar este límite, muchas técnicas para la valoración de los servicios ecosistémicos se han desarrollado y aplicado. Estas técnicas permiten la asignación de un valor a los servicios ecosistémicos sin un mercado, tales como la regulación del clima y el agua, la protección contra los peligros naturales y la biodiversidad.
Desde un punto de vista espacial, los servicios ecosistémicos son heterogéneos a través del paisaje (Fisher et al. 2009). En otras palabras, los servicios ecosistémicos están relacionados con la dimensión espacial de una zona definida en la cual dichos servicios son proporcionados (Busch et al. 2012). Por lo tanto, para una planificación a escala local, es fundamental un conocimiento sólido de la importancia económica y de la distribución espacial de los servicios ecosistémicos. En particular, esta distribución espacial de los servicios ecosistémicos proporciona una información importante a la hora de apoyar a los responsables en la definición y aplicación de las estrategias, que tienen como fin una planificación del paisaje en diferentes partes del territorio.

La gran variedad de enfoques e indicadores utilizados para analizar dicha distribución espacial ha sido documentada de forma detallada por varios trabajos (Maes et al. 2012). El principal enfoque desarrollado a lo largo de los años resalta el dinamismo entre las relaciones causales y las variables ambientales, así como procesos y servicios ecosistémicos más específicos. Este enfoque en función del modelo de relación entre las variables ambientales es probable que produzca mejores resultados, particularmente si está basado en conjuntos de datos iniciales sólidos. No obstante, es necesario logar una adecuada toma de datos y un amplio y experimentado conocimiento. Grêt-Regamey et al. (2013) y Lavorel et al. (2011) han demostrado cómo los modelos de relación entre las variables ambientales pueden ser aplicados en áreas alpinas con éxito.

La pregunta de investigación que fundamenta este estudio es cómo se distribuye espacialmente el valor económico de los diferentes servicios ecosistémicos. El objetivo de este artículo es desarrollar un método que proporcione información detallada y precisa sobre la distribución espacial de los valores correspondientes a los servicios ecosistémicos. Este trabajo forma parte del proyecto recharge. green (Programa Espacial Alpino), en el que la distribución espacial del valor de los servicios ecosistémicos podrá ser utilizada para pronosticar futuros impactos de la aplicación de las energías renovables (por ejemplo, energía hidroeléctrica y biomasa forestal) en algunas áreas de estudio de Los Alpes.

\section{MÉTODOS}

Área de estudio. El área de estudio Gesso-Vermenagna $\left(44^{\circ} 15^{\prime} 00^{\prime \prime} \mathrm{N}, 7^{\circ} 32^{\prime} 00^{\prime \prime} \mathrm{E}\right)$ está localizada en el noroeste de los Alpes Italianos (región del Piemonte, provincia de Cuneo). El área de estudio (figura 1) incluye siete municipios (Valdieri, Entracque, Roaschia, Roccavione, Robilante, Vernante y Limone Piemonte) y cuenta con una población de 10.022 habitantes (densidad = 0,194 habitantes $\left.h^{-1}, 2010\right)$. La superficie del área es de aproximadamente 51.500 ha, de las cuales, alrededor del $62 \%$ están situadas dentro de áreas protegidas (por ejemplo, Parque Natural de Los Alpes Marítimos y Natura 2000). El paisaje del valle Gesso-Vermenagna es un típico paisaje alpino caracteri- 


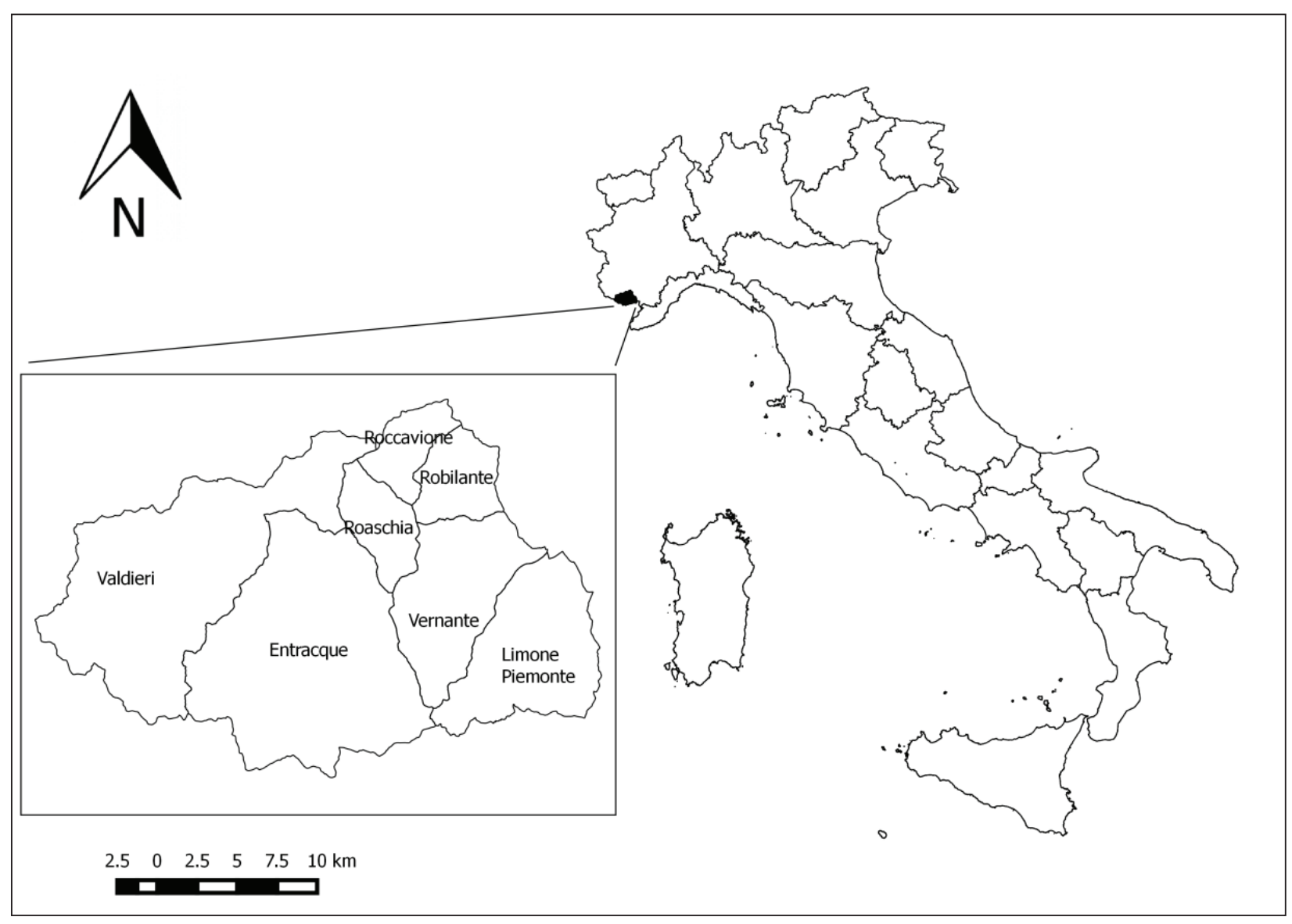

Figura 1. Localización del área de estudio (Gesso-Vermenanga) Italia.

Location of the study area (Gesso-Vermenanga valley, Italy).

zado por un alto grado de biodiversidad (2.600 especies de fauna y flora de las cuales un gran número pertenece a la Lista Roja de Especies Amenazadas de la UICN). Las características morfológicas y litológicas del terreno proporcionan al área de estudio un alto riesgo a los deslizamientos (deslizamientos de tierra y caída de rocas), por este motivo, un alto porcentaje de los bosques está sujeto a limitaciones en la gestión (Ley número 3267 de 1923). El área de estudio es fundamental para el turismo invernal debido a las pistas de esquí localizadas en Limone Piemonte, mientras que en otros municipios de la zona predomina el sector primario (la agricultura de montaña y la gestión forestal). Los principales usos del suelo son forestal (42\%) y pastoral $(33 \%)$, seguidos por tierras improductivas $(21 \%)$ y praderas $(5 \%)$.

Con relación a la propiedad de los bosques, alrededor del $45 \%$ son bosques públicos, mientras que el $55 \%$ restante son bosques de titularidad privada (IPLA 2001). Las especies forestales dominantes son el haya europea (Fagus sylvatica L.) con 11.500 ha de bosque, el castaño (Castanea sativa Mill.) con 2.700 ha, el lárice europeo (Larix decidua Mill.) con 700 ha y el roble pubescente (Quercus pubescens Willd.) con 225 ha. Además del bosque mixto que está conformado por el arce (Acer pseudoplatanus L.), el fresno (Fraxinus ornus L.) y el tilo (Tilia cordata Mill.).

El volumen medio de madera es de $183 \mathrm{~m}^{3} \mathrm{ha}^{-1}$ con algunas diferencias importantes entre los distintos tipos forestales: $245 \mathrm{~m}^{3} \mathrm{ha}^{-1}$ en bosques de castaños, $156 \mathrm{~m}^{3} \mathrm{ha}^{-1}$ en bosques mixtos de arce, fresno y tilo, y $149 \mathrm{~m}^{3} \mathrm{ha}^{-1}$ en bosques de hayas europeas. El incremento del promedio anual es de $7,73 \mathrm{~m}^{3} \mathrm{ha}^{-1}$ por año y con una tasa de cosecha que varía en función de los distintos tipos de especies forestales: $45 \%$ de incremento anual de haya europea y bosques mixtos de latifolios, y el $80 \%$ en bosques de castaños (IPLA 2001).

Evaluación de los servicios ecosistémicos. En una primera etapa del estudio, los principales bienes y servicios ecosistémicos proporcionados por los bosques fueron evaluados desde un punto de vista económico, utilizando las técnicas adecuadas de evaluación ambiental. En particular, en el presente estudio se consideraron tres categorías para los servicios ecosistémicos: (i) servicios de aprovisionamiento, (ii) servicios de regulación y (iii) servicios culturales. 
Los principales beneficios proporcionados por los servicios ecosistémicos forestales han sido identificados por medio del asesoramiento de expertos (Grilli et al. 2015). En particular, se entrevistó a ocho expertos con el fin de poner de relieve los beneficios a escala local. Estos beneficios fueron cuantificados desde un punto de vista monetario, utilizando diferentes métodos de valoración económica, tales como el precio de mercado, el método del costo de reemplazo (método de costo de reposición), y el método de transferencia de beneficios. A continuación, se realizó una estimación de la distribución de dichos beneficios a nivel de paisaje. Los servicios ecosistémicos forestales considerados en este estudio están enumerados en el cuadro 1, junto con los métodos de valoración económica, citados anteriormente. Todos los beneficios suministrados por los servicios ecosistémicos forestales fueron evaluados como flujo anual en referencia al año 2012.

Servicios de aprovisionamiento. Los servicios ecosistémicos más fácilmente reconocibles son los de aprovisionamiento, se trata de bienes tangibles, también llamados recursos naturales o bienes.

En este artículo fueron considerados los servicios como la madera para construcción, los recursos energéticos procedentes del bosque como la leña para combustible y las astillas de madera, los productos forestales no maderables (PFNM) y la provisión de agua proveniente de los bosques.

La energía procedente de la madera y de la leña del bosque, ha sido valorada teniendo en cuenta el precio del mercado local (distinguiendo entre especies y diversidad maderera) y el volumen anual de la cosecha en referencia a los tipos de bosques. El precio local de mercado para la madera varía entre un mínimo de $40 € \mathrm{~m}^{-3}$ para el haya europea y el castaño, y un máximo de $110 € \mathrm{~m}^{-3}$ para el lárice europeo. El incremento anual del volumen maderable es de $6,9 \mathrm{~m}^{3} \mathrm{ha}^{-1}$ año-1 ${ }^{-1}$ mientras que el índice de cosecha es de, aproximadamente, un $20 \%$ del incremento anual. Hoy en día, la producción de astillas de madera que utiliza los residuos forestales (leña de ramas y copas) es de aproximadamente $9.000 \mathrm{~kg} \mathrm{ha}^{-1}$ año $^{-1}$. Esta cantidad es bastante baja en comparación con otras áreas alpinas (por ejemplo Norte y Sur Tirol, Vorarlberg y Trentino) a causa del escaso rendimiento económico (Sacchelli et al. 2013, Paletto et al. 2015): bajo nivel de mecanización, costes elevados del aserrado y también precios de mercado bajos para el aglomerado.

Los PFNM fueron estimados considerando los principales productos no leñosos, tales como los que provienen de la caza (carne, trofeos y pieles), las bayas (arándanos y frambuesas), las setas y las trufas.

El valor económico de los productos procedentes de la caza se calculó a partir de los datos de ungulados cazados durante el año 2012 en el valle del Gesso-Vermenagna (por ejemplo, gamuzas y corzos). Para ello se consideraron tres componentes: la carne, la piel y los trofeos (estos últimos sólo se han tenido en consideración si la pieza cazada es de sexo masculino).

Asimismo, la cantidad de bayas y setas se consideró haciendo referencia a las familias establecidas en el área de estudio (alrededor de unas 4.000 familias). El análisis directo sobre la importancia de la recolección de los PFNM para los habitantes, realizada por Šišak (2006) en la República Checa, muestra que la cantidad media anual de recolección de los PFNM por parte de los propietarios es: setas $5,36 \mathrm{~kg}$ por familia, arándanos 2,61 kg por familia, frambuesas $1,00 \mathrm{~kg}$ por familia. Los datos medios procesados por Šišak (2006) se revisaron sobre la base de los residentes y de la superficie ocupada por bosques en el área de estudio del Gesso-Vermenagna. El valor económico estimado de los PFNM se hizo por hectárea. Además, teniendo en cuenta los datos empíricos sobre las actividades al aire libre por Wilhelmsen (2009) en Noruega, la cantidad de recolección de los PFNM se calculó sólo para un tercio

Cuadro 1. Bienes y servicios de los ecosistemas forestales y los métodos de valoración económica considerados en el estudio.

Forest ecosystem goods and services and economic valuation methods used.

Servicios de aprovisionamiento

Producción de madera

Precio de mercado

Producción leñosa

Precio de mercado

Productos forestales no maderables (productos de caza, bayas, setas, trufas, castañas)

Precio de mercado

Suministro de agua

Precio de mercado

Servicios de regulación

Protección contra riesgos naturales (protección directa e indirecta)

Método del costo de reemplazo

Almacenamiento de carbono

Precio de mercado voluntario

Servicios culturales

Recreación al aire libre (excursionismo, senderismo, etc.)

Método de transferencia de beneficios 
de las familias. En este estudio se consideró que el enfoque utilizado en la República Checa y Noruega es transferible al área de estudio del Gesso-Vermenagna, ya que los tipos de bosques, las especies de setas y la accesibilidad de los residentes en el bosque son comparables.

El valor económico de los PFNM en la región del Piemonte, se calculó multiplicando las cantidades descriptas por los precios locales $\left(10 € \mathrm{~kg}^{-1}\right.$ tanto para las setas como para las bayas). En cuanto a las trufas, se calculó teniendo en cuenta los precios del mercado local, que iban desde los $2 € \mathrm{~g}^{-1}$ de la trufa blanca a los $0,7 € \mathrm{~g}^{-1}$ de la trufa negra y la cantidad media de los productos recolectados con finalidad comercial.

Por último, los bosques influyen en las redes hidrográficas y en el funcionamiento de los ciclos hidrológicos, afectando al suministro de agua tanto para uso doméstico como para uso industrial. El suministro de agua fue evaluado considerando el consumo medio anual de agua per capita $\left(63,9 \mathrm{~m}^{3}\right.$ año-1 por familia) y el precio medio del agua a nivel local $\left(0,98 € \mathrm{~m}^{-3}\right)$. El consumo medio anual de agua ha sido calculado teniendo en cuenta todos los usos locales del agua (usos domésticos, agrícolas, energéticos e industriales); mientras que el precio refleja los costes procedentes del suministro de agua, de la red hídrica y limpieza de la misma.

Servicios de regulación. En esta categoría se incluyen procesos ecosistémicos complejos mediante los cuales se regulan las condiciones del ambiente en que los seres humanos realizan sus actividades productivas.

En este caso, se han tenido en cuenta los beneficios procedentes del almacenamiento de carbono y de la protección contra los riesgos hidrogeológicos procedentes de los servicios ecosistémicos. El almacenamiento de carbono en los bosques se calculó siguiendo el método For-Est (Federici et al. 2008) basado en el Intergovernmental Panel on Climate Change (IPCC 2003). Para poder cuantificar el incremento anual del carbono almacenado en el bosque, se consideraron dos depósitos de carbono, la biomasa aérea y la biomasa subterránea. El carbono almacenado en la biomasa se multiplicó por el costo del mercado voluntario de carbono de 4,59€ $\mathrm{Mg}^{-1}$ de $\mathrm{CO}_{2}$ (Peter-Stanley y Yin 2013). Con el fin de estimar el valor económico del almacenamiento de carbono en la biomasa forestal viva, tanto aérea como subterránea, se empleó la siguiente fórmula [1]:

$$
V_{c}=[(I \cdot B E F \cdot W B D)+(I \cdot W B D \cdot R)] \cdot 0.5 \cdot 3.67 \cdot p_{c}
$$

Donde:

$V_{c}=$ valor del almacenamiento de carbono en la biomasa aérea y subterránea $(€)$;

$I=$ incremento del volumen anual $\left(\mathrm{m}^{3} \mathrm{ha}^{-1}\right.$ año $\left.^{-1}\right)$;

$B E F=$ factor de expansión de la biomasa (generalmente el volumen del bosque está referido al volumen de los troncos, y el factor de expansión representa componentes tales como ramas y hojas);
$W B D=$ densidad básica de la madera;

$R=$ relación raíz/tallo;

$0,5=$ coeficiente de carbono contenido;

$3,67=$ coeficiente de carbono a $\mathrm{CO}_{2}$;

$p_{c}=$ precio del carbono en el mercado voluntario de carbono $\left(€ \mathrm{Mg}^{-1}\right.$ de $\left.\mathrm{CO}_{2}\right)$

La protección contra los riesgos hidrogeológicos, tales como la erosión del suelo, los deslizamientos de tierra y las avalanchas fue estimada mediante el enfoque del método del costo de reemplazo (Notaro y Paletto 2012). Este procedimiento considera el costo incurrido para reemplazar la funcionalidad en los bosques con obras de ingeniería ambiental. Las obras de ingeniería ambiental se escogieron distinguiéndose entre protección directa e indirecta, y teniendo en consideración los diferentes riesgos naturales más importantes del área de estudio (Notaro y Paletto 2012). La protección indirecta puede ser definida como la prevención de la erosión del suelo y la regulación del flujo del agua, mientras que la protección directa implica el salvaguardar la vida y las actividades humanas de los riesgos naturales.

Para aplicar el método del costo de reemplazo, se emplearon los precios de las obras de ingeniería ambiental pública de la región del Piemonte. A fin de calcular el costo anual por unidad de superficie en hectáreas, se consideraron los costos totales de la realización y del mantenimiento de las diferentes obras de ingeniería ambiental, haciendo una distinción entre los tipos de protección (directa e indirecta). Para la protección indirecta se consideraron la erosión del suelo y las riberas de los ríos, mientras que para el caso de protección directa se consideraron los deslizamientos de tierra y las avalanchas (cuadro 2). El valor económico de la protección contra los riesgos hidrogeológicos se calculó mediante la siguiente fórmula [2] (Notaro y Paletto 2012):

$$
V_{p}=\frac{u C \cdot r}{(1+r)^{t}}+M
$$

Donde:

$V_{p}=$ valor de la función de protección contra los riesgos hidrogeológicos $(€)$

$u C=$ coste unitario de las obras de ingeniería ambiental $\left(€ \mathrm{~m}^{-2}\right)$

$r=$ tasa de interés $(2 \%)$

$t=$ vida útil de las obras de ingeniería ambiental (años)

$M=$ costes de mantenimiento $(€)$

Para el cálculo anual del costo, se escogió un tipo de interés conservador y se fijó posteriormente al $2 \%$, de acuerdo con el rango establecido por Freeman (2003).

Servicios culturales. Los ecosistemas brindan beneficios que dependen de las percepciones colectivas de los humanos acerca de los ecosistemas y de sus componentes. Los 
Cuadro 2. Obras de ingeniería ambiental utilizadas en sustitución de los bosques con el fin de desarrollar la función de protección contra los riesgos naturales.

Environmental engineering works used as substitute of forests to protect against natural hazards.

\begin{tabular}{lclrc}
\hline \multicolumn{1}{c}{ Tipo de protección } & $\begin{array}{c}\text { Superficie } \\
\text { (ha) }\end{array}$ & \multicolumn{1}{c}{ Obras de ingeniería } & $\begin{array}{c}\text { Costo unitario } \\
\left(€ \mathrm{~m}^{-2}\right)\end{array}$ & $\begin{array}{c}\text { Vida útil } \\
(\mathrm{años})\end{array}$ \\
\hline Erosión del suelo (protección indirecta) & 4,088 & Hidrosiembra & 3,82 & 15 \\
Riberas de los ríos (protección indirecta) & 50,60 & Geotextiles & 14,65 & 20 \\
Deslizamientos de tierra (protección directa) & 256,69 & Emparrillado vivo & 92,56 & 25 \\
Avalanchas (protección directa) & 810,26 & $\begin{array}{l}\text { Estructura en forma de rastrillo } \\
\text { para retener la nieve }\end{array}$ & 265,30 & 25 \\
\hline
\end{tabular}

servicios culturales provenientes de los bosques pueden ser definidos como los beneficios no materiales que las personas obtienen de los mismos, a través de un enriquecimiento espiritual, de un desarrollo cognitivo o de la recreación y experiencia estética (Maes et al. 2012). En este estudio, se consideró la recreación y experiencia estética, con especial énfasis en las actividades que se realizan al aire libre como son el senderismo, el excursionismo, trekking.

Las actividades al aire libre fueron evaluadas mediante el método de transferencia de beneficios (BT, benefit transfer). Dicho método consiste en la adaptación de información, derivada desde una investigación original, para la aplicación de ésta en un contexto diferente de estudio. La transferencia de beneficios comprende la transferencia del beneficio económico estimado de un sitio, donde ya se ha llevado a cabo un estudio ("sitio de origen"), al sitio donde se tomarán decisiones de manejo de recursos naturales ("sitio de política").

El valor económico estimado de un "sitio de estudio" puede ser transferido a un "sitio de política" ya sea como unidades monetarias (transferencia de valores), o como una función (transferencia de funciones), que define un escenario de elección económico y ecológico (Rosenberger y Loomis 2001).

El valor promedio utilizado por el método de transferencia toma la medida de la tendencia central de todos los estudios analizados "sitio de estudio" y lo transfiere al "sitio de política".

En este estudio se utilizó el método de transferencia de valor promedio para ambos servicios recreacionales, tomando los resultados obtenidos por Grilli et al. (2014) para los bosques de montaña en Europa ("sitios de estudio"). La recreación al aire libre ha sido evaluada a partir de estudios disponibles en la literatura, se recogieron y posteriormente analizaron 32 documentos publicados entre 1977 y 2013. Por otro lado, los valores de la recreación al aire libre obtenidos de los meta-análisis, se transfirieron de acuerdo con los tipos forestales (bosques mixtos, bosques de coníferas puras y bosques de latifolias puras) y con el flujo turístico por municipio.

Los valores de recreación al aire libre, provenientes de los meta-análisis, se calcularon para el año 2012 utilizando la tasa social de descuento (TSD) del $1 \%$. La tasa social de descuento es el precio sombra que se atribuye al capital para evaluar la inversión pública. Esta tasa depende de la utilidad social de la inversión pública y puede ser elegido entre $0,5 \%$ y $2 \%$ (Freeman 2003).

Enfoque espacial. En la segunda etapa del estudio, se analizó la distribución espacial de los valores de los servicios ecosistémicos para el caso de estudio del Valle del GessoVermenagna (Alpes Italianos) a través de un sistema de información geográfica (SIG), teniendo en consideración las características ecológicas de cada servicio ecosistémico. La creación del mapa de los beneficios procedentes de los servicios ecosistémicos se realizó teniendo en consideración las características ecológicas de cada servicio ecosistémico. Cada servicio ecosistémico fue valorado de manera individualizada otorgándoles un valor económico y usando datos georeferenciados con el fin de obtener un valor económico único para cada porción del territorio. Para el análisis espacial, se utilizaron los software Quantum GIS y ArcGIS.

Con el fin de crear el mapa espacial de los servicios ecosistémicos se utilizó un conjunto de capas temáticas que representan las variables clave del estudio (figura 2). Estas capas temáticas fueron proporcionadas por la Región del Piemonte. Para ello, las capas fueron superpuestas con el fin de analizar la distribución espacial que presentan los distintos beneficios. Las capas temáticas utilizadas fueron: (1) uso del suelo, (bosques, pastizales, campos, cultivos agrícolas y asentamientos); (2) especies forestales (bosques de coníferas puras, bosques de latifolias puras y bosques mixtos); (3) límites municipales; (4) redes hidrográficas (ríos y arroyos); (4) tipos de protección forestal (protección directa o indirecta). En particular, los ríos y arroyos fueron representados mediante el diseño de un buffer con una distancia de referencia de $5 \mathrm{~m}$, y los bosques de protección fueron representados mediante capas temáticas específicas.

Asimismo, se utilizó los diferentes usos de la tierra para distinguir las áreas que han sido evaluadas dentro de los bosques del área de estudio. El mapa resultante se caracterizó por un número de polígonos que muestran los valores suministrados por los servicios ecosistémicos. 


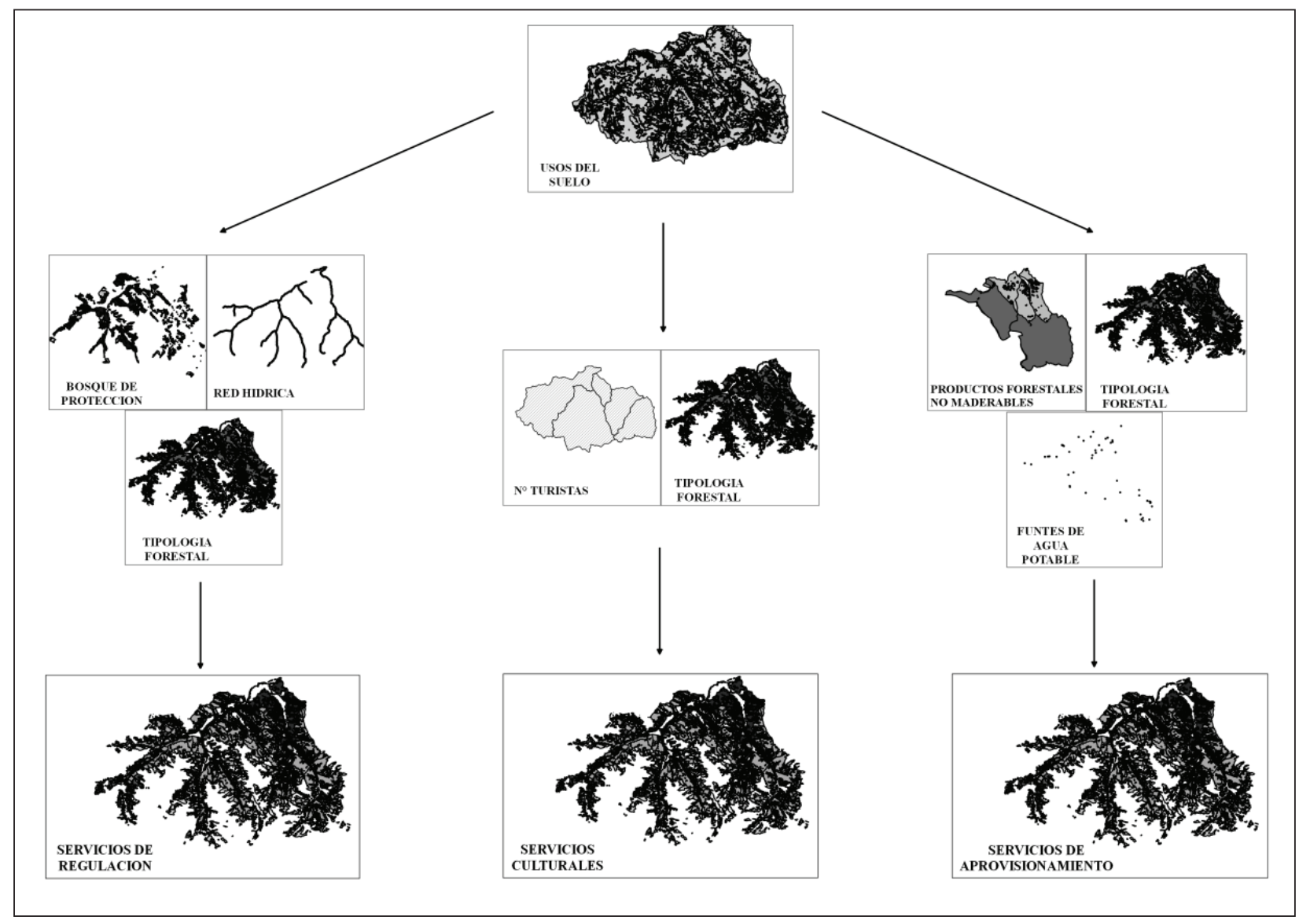

Figura 2. Representación gráfica del marco metodológico para la creación de la distribución espacial de los servicios ecosistémicos. Method used for mapping forest ecosystem services.

\section{RESULTADOS}

Servicios de aprovisionamiento. El valor total de la producción de madera es aproximadamente de $131 €$ ha $^{-1}$ año ${ }^{-1}$, cuyo valor estuvo subdividido en $79 €$ ha $^{-1}$ por año de madera para construcción, $41 €$ ha $^{-1}$ año ${ }^{-1}$ de leña para combustible, y $11 €$ ha $^{-1}$ año ${ }^{-1}$ de astillas de madera. Los valores medios de la producción de madera, distintos para los tipos de masas forestales, varían entre un máximo de 250 $€$ ha $^{-1}$ año ${ }^{-1}$ para los bosques de abeto blanco a un mínimo de $10 €$ ha $^{-1} a_{n} o^{-1}$ para los bosques de roble pubescente. La producción de astillas de madera principalmente se centra en tres tipos de masas forestales: bosques de haya europea $\left(21 €\right.$ ha $^{-1}$ año $\left.{ }^{-1}\right)$, bosques de roble pubescente (14€ $\mathrm{ha}^{-1}$ año $\left.^{-1}\right)$ y bosques de castaño $\left(5 € \mathrm{ha}^{-1}\right.$ año $\left.^{-1}\right)$.

Todos los PFNM a excepción de las castañas tuvieron una importancia económica relativamente baja: las setas $4,0 €$ ha $^{-1}$ año $^{-1}$, las trufas $2,8 €$ ha $^{-1}$ año $^{-1}$, los arándanos $2,0 €$ ha $^{-1}$ año ${ }^{-1}$, los productos de caza $1,8 €$ ha $^{-1}$ año ${ }^{-1}$, y las frambuesas $0,8 €$ ha $^{-1}$ año $^{-1}$. Desde un punto de vista económico, las castañas representan la fuente de aprovisionamiento más importante, con valores superiores a $1.900 €$ $\mathrm{ha}^{-1} \mathrm{año}^{-1}$. Este hecho se debe a una importante tradición en la producción de castañas en la región del Piemonte.

En el valle del Gesso-Vermenagna, el suministro del agua tiene aproximadamente un valor económico de $250.000 €$ año $^{-1}\left(11,7 €\right.$ ha $^{-1}$ año $\left.^{-1}\right)$.

Los resultados de la valoración económica de los servicios de aprovisionamiento del valle de Gesso-Vermenagna (figura 3) evidencian valores finales medio-altos, si bien estos, están concentrados en algunas áreas particulares del valle. Los ecosistemas forestales se caracterizan por tener una buena capacidad productiva. En particular, el mercado local de la leña en esta área de estudio se destina predominantemente para combustible y a las astillas de madera, mientras que el uso de la madera está bastante limitado debido a la escasa presencia de aserraderos en la zona.

La figura 3 muestra, además, que los valores más elevados se observan en los bosques a una altura más baja del valle de Vermenagna, concretamente en los municipios de Rocabilante, Roccavione y Roaschia. En esta área se concentran las superficies forestales principalmente productivas, atribuibles a las presencia de las hayas, de los castaños y de los fresnos, tilos, y arces. Para la economía local el 


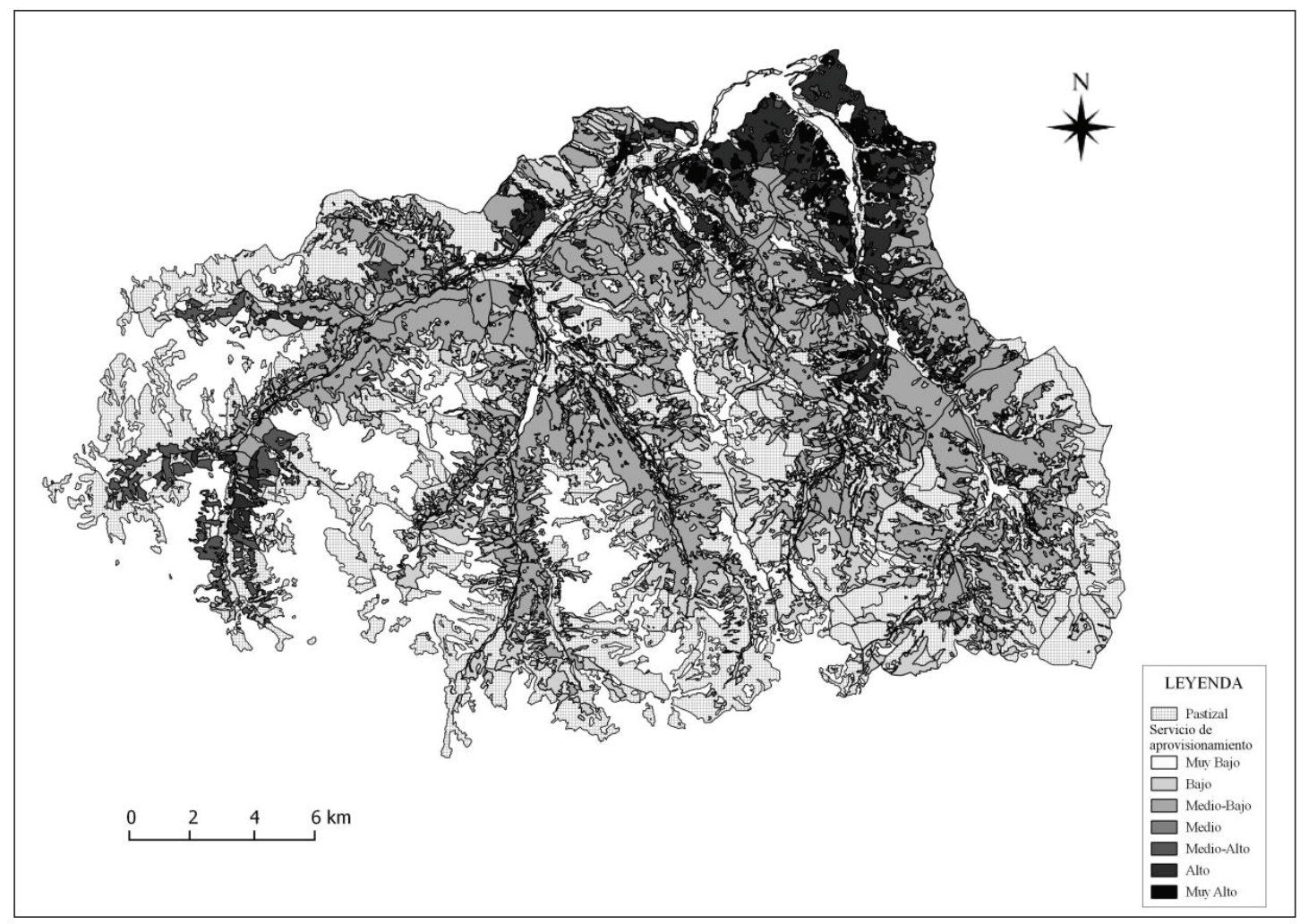

Figura 3. Distribución de los valores económicos relativos a los servicios de aprovisionamiento en el valle del Gesso-Vermenanga. Spatial distribution of the economic values of provisioning services in Gesso-Vermenagna valley.

cultivo de castaños es de vital importancia. Los castaños de fruto con el precio más elevado se concentran en las comunidades de Robilante y Roccavione presentando las áreas con mayor valor.

Servicios de regulación. Los resultados del presente estudio muestran que estas funciones tuvieron un valor de $3.765 € \mathrm{ha}^{-1} \mathrm{año}^{-1}$ para la protección contra las avalanchas, $568 € \mathrm{ha}^{-1}$ año $^{-1}$ para la protección contra la erosión del suelo (protección indirecta), y un valor de $707 € \mathrm{ha}^{-1}$ año $^{-1}$ para la protección directa contra los deslizamientos de tierra.

Los resultados muestran que la cantidad de almacenamiento de carbono es de $2,88 \mathrm{Mg}$ ha por año, de la cual $2,47 \mathrm{Mg}$ ha $^{-1}$ corresponden a la biomasa aérea y $0,41 \mathrm{Mg} \mathrm{ha}^{-1}$ a la biomasa subterránea, correspondiente a un valor promedio de $48 € \mathrm{ha}^{-1} \mathrm{año}^{-1}$. Los valores medios distintos para los tipos de masas forestales varían entre un máximo de $61 €$ ha $^{-1}$ año ${ }^{-1}$ para los bosques de roble pubescente a un mínimo de $10 €$ ha $^{-1}$ año $^{-1}$ para los bosques mixtos de sauce y álamo.

En relación con los servicios de regulación cabe destacar que los bosques del Valle de Gesso-Vermenagna tienen un papel importante en el almacenamiento del carbono, en especial algunas formaciones forestales como los bosques mixtos y los bosques de coníferas puras.

Por otro lado, una gran parte de los bosques del valle de Gesso-Vermenagna mantienen una función de protec- ción importante contra los riesgos naturales debido a la morfología del territorio, especialmente contra los deslizamientos de tierra y las avalanchas. En la figura 4 se evidencia el alto valor económico de los bosques de protección, que resulta ser muy elevado respecto a los bosques de protección directa (riesgo de avalanchas) en las comunidades de Entracque, Valdieri y Limone Piemonte.

Servicios culturales. Los resultados de la recreación al aire libre muestran diferencias interesantes debido a las características del paisaje y a los flujos turísticos. El valor promedio de los servicios generados por la recreación al aire libre en los bosques del Gesso-Vermenagna es de 26,1€ $\mathrm{ha}^{-1}$ año ${ }^{-1}$ con un rango que comprende los $95,4 € \mathrm{ha}^{-1}$ año ${ }^{1}$ para los bosques mixtos y los $24,5 € \mathrm{ha}^{-1}$ año $^{-1}$ para los bosques de latifolias puras.

En la figura 5 se puede apreciar cómo el municipio del Limone Piemonte, situado al sureste del mapa, presenta un valor significativamente superior que en las áreas restantes debido a los elevados flujos turísticos relacionados principalmente con las actividades invernales. El flujo de turistas en el municipio de Limone Piemonte fue el más elevado (aproximadamente 66.900 turistas anuales), mientras que en otros municipios (Entraque, Valdieri y Vernante) destaca el fenómeno del eco-turismo (aproximadamente 20.000 turistas anuales). Mientras que las zonas del fondo del va- 


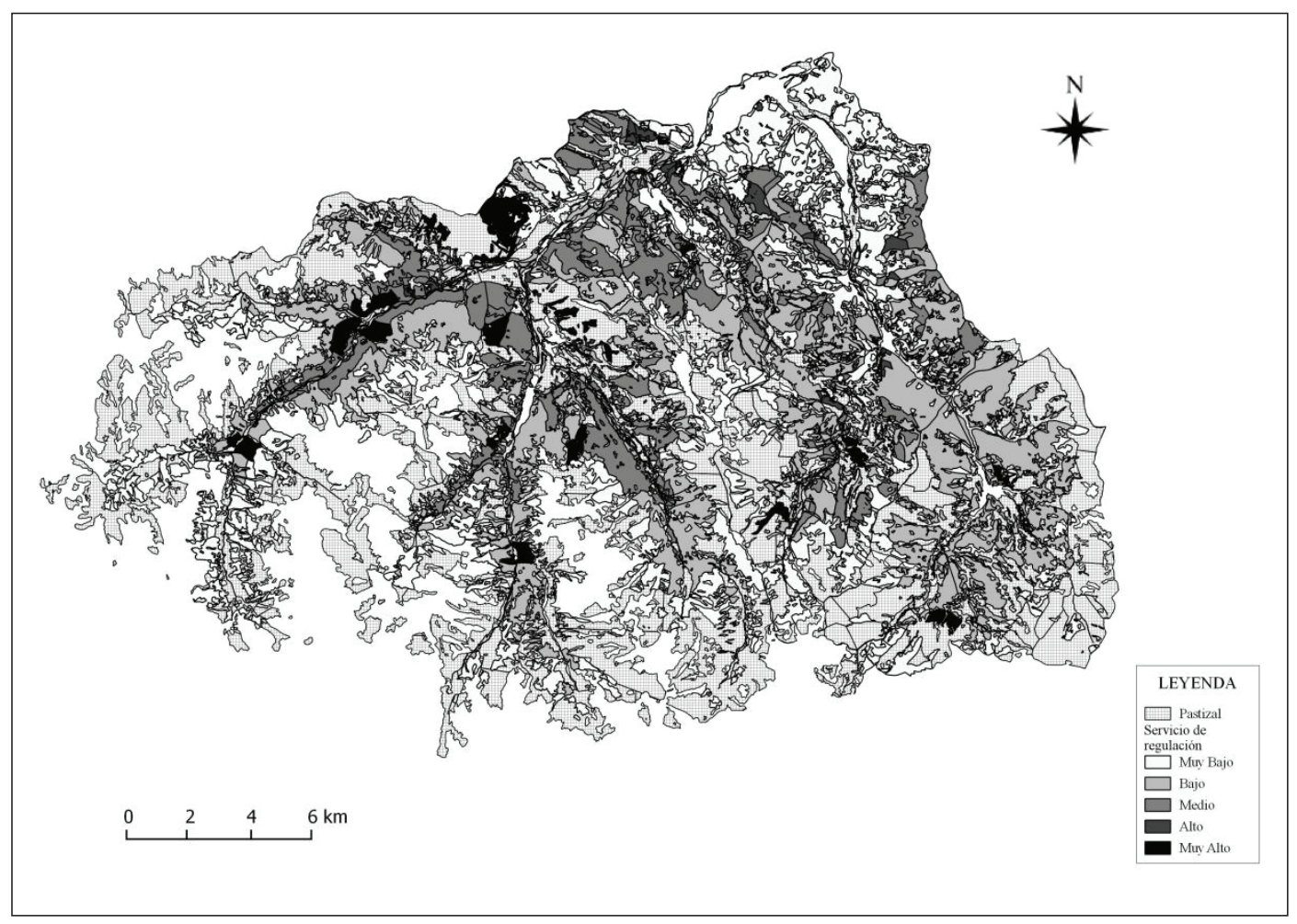

Figura 4. Distribución de los valores económicos relativos a los servicios de regulación en el valle del Gesso-Vermenanga. Spatial distribution of the economic values of the regulating services in Gesso-Vermenagna valley.

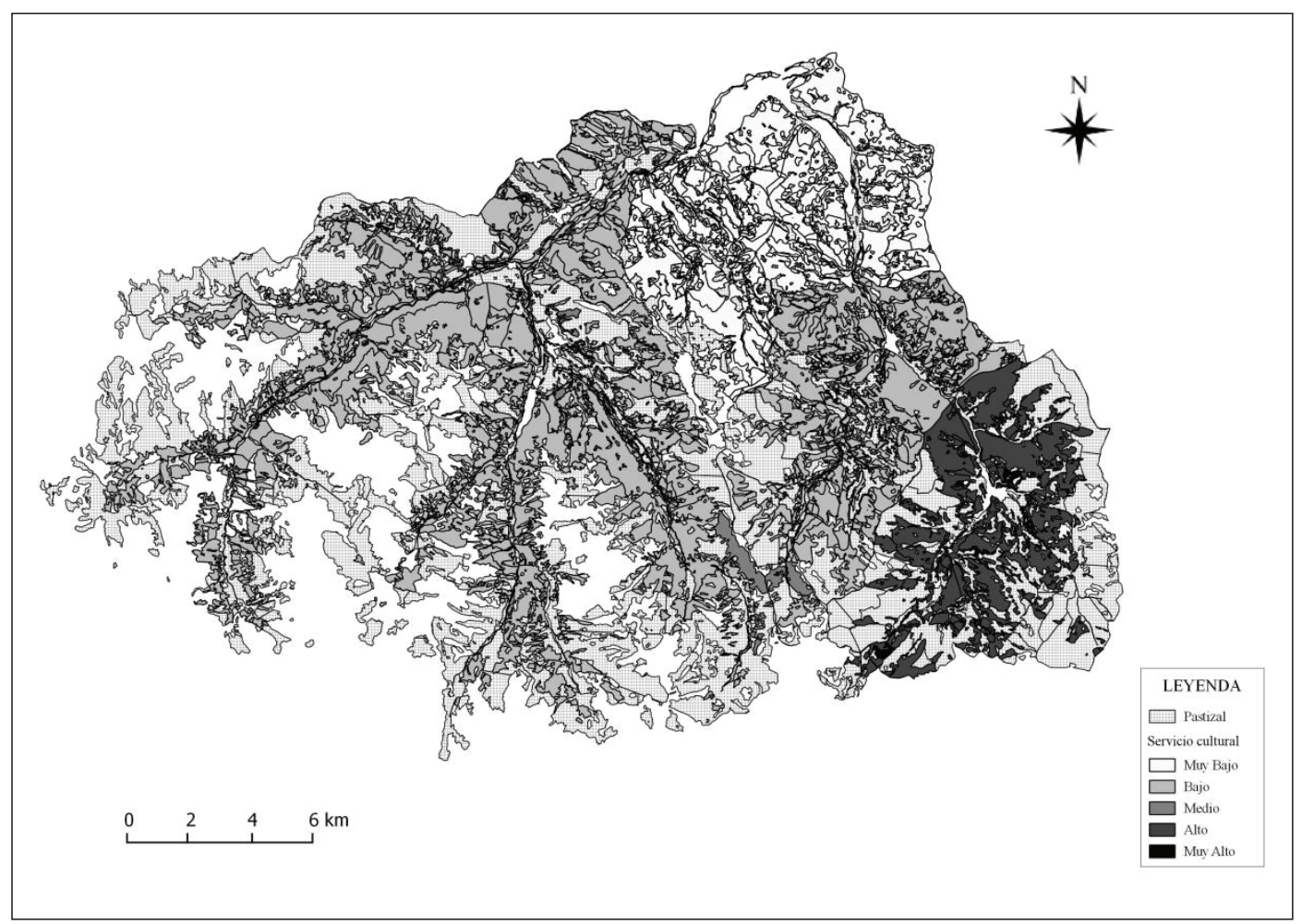

Figura 5. Distribución de los valores económicos relativos a los servicios culturales en el valle del Gesso-Vermenanga. Spatial distribution of the economic values of cultural services in Gesso-Vermenagna valley. 
lle, los municipios de Roaschia, Roccavione y Robilante, presentan un bajo nivel de atracción turística. Las áreas de los municipios de Entracque y Valdieri (correspondientes al territorio del Parque Natural de Los Alpes Marítimos) poseen a su vez un flujo turístico medio-bajo, si se compara con el del Limone Piemonte.

Por último, se puede afirmar que el desequilibrio espacial en el flujo turístico del valle del Gesso-Vermenanga se debe principalmente a la presencia de una estación de esquí de relevancia internacional en el territorio del municipio de Limone Piemonte y a un túnel (túnel de Tenda) que permite el tránsito entre Italia y Francia durante todo el año. Estos aspectos son los que condicionan la distribución de los valores económicos relativos a los servicios culturales. En particular, el valor económico de los servicios culturales está fuertemente ligado al flujo turístico, zonas muy concurridas por los turistas poseen valores significativamente más altos que las zonas menos concurridas. Por lo tanto, las zonas con presencia de equipamientos turísticos (hoteles, pistas de esquí) poseen valores más altos que las zonas frecuentadas solamente por excursionistas.

\section{DISCUSIÓN}

Servicios de aprovisionamiento. Los resultados muestran que los servicios de aprovisionamiento, tales como la madera para construcción y los recursos energéticos procedentes del bosque (como la leña para combustible y las astillas de madera) no han tenido una alta importancia económica en el valle del Gesso-Vermenagna. Este valor es bajo porque la producción de biomasa forestal para uso energético es aún limitada debido al bajo nivel de mecanización de las empresas forestales locales. Por otra parte, la morfología de la zona dificulta la extracción de biomasa forestal.

Por otra parte, el valor económico del suministro del agua es bastante bajo en comparación con el estudio realizado por Getzner y Policy (2010), el cual analizó el valor de los servicios ecosistémicos en el Parque Nacional del Tatra (Polonia). El valor económico de este servicio está estrechamente vinculado al precio de mercado del agua. El precio varía considerablemente de un país a otro y en función del tipo de uso del agua (agua de uso doméstico, agua en la agricultura, agua para uso industrial y agua para la producción de energía). En referencia a los países europeos el precio de mercado del agua potable en 2010 fue menor que 2 US\$ $\mathrm{m}^{-3}$ en Grecia, Italia y Portugal y fue superior a 6 US\$ $\mathrm{m}^{-3}$ en Dinamarca (OECD 2010).

Servicios de regulación. Los resultados del presente estudio confirman la gran importancia que tienen los bosques en cuanto concierne a servicios de regulación. En áreas montañosas, la protección de los bosques contra los riesgos naturales es considerada como el servicio ecosistémico más importante desde un punto de vista tanto económico como ecológico (Merlo y Rojas Briales 2000). Las diferencias en los valores de la función de protección no están re- lacionadas con los tipos de masas forestales. Es importante destacar que los valores económicos, que hacen referencia a la protección hidrogeológica de los bosques alpinos, se han evidenciado en otras áreas de estudio con resultados comparables a los obtenidos en el presente estudio. Goio et al. (2008) mostraron un valor promedio de 212,2€ ha-1 año ${ }^{-1}$ para toda la provincia de Trento, mientras que Notaro y Paletto (2012) en un bosque a pequeña escala (Bosque de Valdastico) evidenciaron un valor de 284,2 € ha-1 año-1 para la función de protección hidrogeológica. Por último, se ha destacado que la evaluación de estos servicios ecosistémicos está sumamente influenciada por el método de valoración económica que ha sido utilizado.

El valor promedio de almacenamiento de carbono es similar al estimado en otros casos de estudio europeos donde los valores de biomasa aérea varían entre 6 y $40 €$ $\mathrm{ha}^{-1} \mathrm{año}^{-1}$ (Goio et al. 2008, Hein 2011). Asimismo, un estudio en España que analizó la voluntad de la población a pagar la forestación de tierras agrícolas marginales, que reducirían el dióxido de carbono en la atmósfera, muestra un valor comprendido entre $0,0004 €$ y $0,025 € \mathrm{Mg}^{-1}$ de $\mathrm{CO}_{2}$ por persona al año (Mavsar et al. 2014).

Servicios culturales. En este estudio, el valor promedio de la recreación al aire libre se encuentra dentro del rango de los valores evidenciados por otros estudios en Europa, no obstante la utilización de diferentes métodos de valoración económica (Goio et al. 2008, Bartczak et al. 2008, Voces González et al. 2010). Zandersen y Tol (2009) muestran un amplio rango de valores que van desde los 0,66€ a los $112 €$ por visita, con especial referencia a 26 estudios europeos de recreación forestal desde 1979 a 2001 . Esta elevada variabilidad se encuentra dentro de otros factores relacionados con los métodos de valoración económica utilizados (por ejemplo, la valoración contingente, el método del coste del viaje, la elección del experimento), el atractivo turístico de la zona (por ejemplo, las áreas protegidas), la distancia de las zonas urbanas, y las características socioeconómicas de los turistas (las diferencias culturales).

\section{CONCLUSIONES}

Como conclusión se puede afirmar que el método propuesto permite georreferenciar los valores de los servicios ecosistémicos teniendo en cuenta algunas de las variables espaciales de simple adquisición y elaboración, tales como el uso del suelo, el tipo forestal y la actividad turística de la zona. La distribución espacial del valor de cada uno de los servicios ecosistémicos permite ampliar el conocimiento y la información útil para los responsables encargados de la toma de decisiones (por ejemplo, responsables políticos, gestores de los recursos naturales) con el fin de minimizar los impactos negativos sobre el medio ambiente. Además, un conocimiento detallado de la distribución espacial de cada uno de los servicios ecosistémicos erogados de los bosques permite programar las intervenciones de la ges- 
tión forestal que tienen como finalidad máxima la producción leñosa, intentando de minimizar al mismo tiempo los impactos negativos sobre otros servicios (de regulación y culturales). Tener en consideración este aspecto en la fase preliminar del trabajo permite evitar el doble cálculo desde el punto de vista económico y los potenciales errores en el mapeo de los datos. Por otra parte, la distribución espacial del valor económico de los servicios ecosistémicos permite evaluar con un alto grado de precisión los impactos ecológicos de la aplicación de energías renovables. En particular, la extracción de biomasa forestal puede causar impactos sobre la biodiversidad (por ejemplo: los insectos saproxílicos que dependen de la cantidad de madera muerta), la fertilidad del suelo y la regulación del agua, mientras que la producción de energía hidroeléctrica puede causar impactos negativos sobre la biodiversidad del río. La evaluación ecológica y económica de los servicios ecosistémicos puede permitir una mejor planificación de las intervenciones que limiten el impacto sobre el medio ambiente. Por ejemplo, algunas áreas importantes para la protección del suelo fueron excluidas de la extracción de biomasa forestal, mientras que en otras zonas de alto valor turístico y de recreación se decidió eliminar totalmente los residuos de madera.

Los puntos fuertes del método propuesto han sido la simplicidad de los métodos utilizados para evaluar de manera individual los servicios ecosistémicos, el número limitado de datos requeridos para realizar la localización espacial de los servicios ecosistémicos, y la facilidad con la que el método puede ser reproducido en otros contextos geográficos. Por el contrario, el punto débil del método sugerido es típico de las valoraciones económicas, como por ejemplo, la elevada variabilidad entre los beneficios de los servicios ecosistémicos que derivan de diferentes estudios. Esta variabilidad se debe a las características de las zonas y a los métodos de valoración económica utilizados.

\section{REFERENCIAS}

Bartczak A, H Lindhjem, A Stenger. 2008. Review of benefit transfer studies in the forest context. Scandinavian Journal of Forest Research 42:276-304.

Busch M, A La Notte, V Laporte, M Erhard. 2012. Potentials of quantitative and qualitative approaches to assessing ecosystem services. Ecological Indicators 21:89-103.

Costanza R, R d'Arge, R de Groot, S Farberk, M Grasso, B Hannon, K Limburg, S Naeem, RV O’Neill, J Paruelo, RG Raskin, P Suttonkk, M van den Belt. 1997. The value of the world's ecosystem services and natural capital. Nature 387:253-260.

De Groot R, R Alkemade, L Braat, L Hein, L Willemen. 2010. Challenges in integrating the concept of ecosystem services and values in landscape planning, management and decision making. Ecological Complexity 7:260-272.

Eade DOE, D Moran. 1996. Spatial economic valuation: benefit transfer using Geographical Information Systems. Journal of Environmental Management 48:97-110.
Federici S, M Vitullo, S Tulipano, R De Lauretis, G Seufert. 2008. An approach to estimate carbon stocks change in forest carbon pools under the UNFCCC: the Italian case. iForest 1(1):86-95.

Fisher B, RK Turner, P Morling. 2009. Defining and classifying ecosystem services for decision making. Ecological Economics 68(3):643-653.

Freeman AM. III 2003. The Measurement of Environmental and Resource Values: Theory and Methods, 2nd edition. Washington, USA. Resources for the Future. 516 p.

Getzner M, I Policy. 2010. Ecosystem services, financing, and the regional economy: A case study from Tatra National Park, Poland. Biodiversity Conservation 11(2):56-61.

Goio I, G Gios, C Pollini. 2008. The development of forest accounting in the province of Trento (Italy). Journal of Forest Economics 14(3):177-196.

Grêt-Regamey A, A Walz, P Bebi. 2008. Valuing ecosystem services for sustainable landscape planning in Alpine regions. Mountain Research and Development 28(2):156-165.

Grêt-Regamey A, SH Brunner, J Altwegg, M Christen, P Bebi. 2013. Integrating expert knowledge into mapping ecosystem services trade-offs for sustainable forest management. Ecology and Society 18(3):34.

Grilli G, G Curetti, I De Meo, G Garegnani, F Miotello, A Poljanec, D Vettorato, A Paletto. 2015. Experts' perceptions of the effects of forest biomass harvesting on sustainability in the Alpine region. South-East European Forestry 6(1):77-95.

Grilli G, A Paletto, I De Meo. 2014. Economic Valuation of Forest Recreation in an Alpine valley. Baltic Forestry 20(1):1-23.

Hein L, K van Koppen, RS de Groot, EC van Ierland. 2006. Spatial scales, stakeholders and the valuation of ecosystem services. Ecological Economics 57(2):209-228.

Hein L. 2011. Economic benefits generated by protected areas: the case of the Hoge Veluwe forest, the Netherlands. Ecology and Society 16(2):13.

IPCC (Intergovernmental Panel on Climate Change, CH). 2003. Good Practice Guidance for Land Use, Land Use Changes and Forestry. Kanagawa, Japan. IPCC. 584 p.

IPLA (Istituto per le Piante da Legno e l'Ambiente, IT). 2001. Area forestale: Valli Gesso, Vermenagna e Pesio. Piano Forestale Territoriale. Torino, Istituto per le Piante da Legno e l'Ambiente, Regione Piemonte.

Lavorel S, K Grigulis, P Lamarque, MP Colace, D Garden, J Girel, G Pellet, R Douzet. 2011. Using plant functional traits to understand the landscape distribution of multiple ecosystem services. Journal of Ecology 99:135-147.

Maes J, B Egoh, L Willemen, C Liquete, P Vihervaara, JP Schägner, B Grizzetti, EG Drakou, A La Notte, G Zulian, F Bouraoui, ML Paracchini, L Braat, G Bidoglio. 2012. Mapping ecosystem services for policy support and decision making in the European Union. Ecosystem Services 1(1):31-39.

Mavsar R, E Varela, D Pettenella, S Elizabeth, V Bredahl Jacobsen. 2014. The value of carbon sequestration. In Thorsen J, R Mavsar, L Tyrväinen, I Prokofieva, A Stenger eds. The Provision of Forest Ecosystem Services. Joensuu, Finland. European Forest Institute (EFI). p. 56-62.

MEA (Millennium Ecosystem Assessment, US). 2005. Ecosystems and Human Well-Being: Biodiversity Synthesis. Washington, USA. World Resources Institute. 155 p.

Merlo M, E Rojas Briales. 2000. Public goods and externalities linked to Mediterranean forests: economic nature and poli- 
cy. Land Use Policy 17(3):197-208.

Notaro S, A Paletto. 2012. The economic valuation of natural hazards in mountain forests: An approach based on the replacement cost method. Journal of Forest Economics 18(4):318-328.

OECD (Organisation for Economic Co-operation and Development, FR). 2010. Pricing Water Resources and Water and Sanitation Services. Paris, France. OECD Publishing. 104 p.

Paletto A, C Geitner, G Grilli, R Hastik, F Pastorella, L Rodrìguez Garcìa. 2015. Mapping the value of ecosystem services: A case study from the Austrian Alps. Annals of Forest Research 58(1):157-175.

Peters-Stanley M, D Yin. 2013. Maneuvering the mosaic. State of the voluntary carbon markets 2013. Washington, USA. Ecosystem Marketplace and Bloomberg New Energy Finance. $126 \mathrm{p}$.

Rosenberger RS, JB Loomis. 2001. Benefit transfer of outdoor recreation use values: a technical document supporting the
Forest Service Strategic Plan (2000 revision). General Technical Report. RMRS-GTR-72. Fort Collins, USA. USDA Forest Service, Rocky Mountain Research Station. 59 p.

Sacchelli S, I De Meo, A Paletto. 2013. Bioenergy production and forest multifunctionality: a trade-off analysis using multiscale GIS in a case study in Italy. Applied Energy 104:10-20.

Šišák L. 2006. Importance of non-wood forest product collection and use for inhabitants in the Czech Republic. Journal of Forest Science 52(9):417-426.

Voces González R, L Díaz Balteiro, E López-Peredo Martínez. 2010. Spatial valuation of recreation activities in forest systems: application to province of Segovia (Spain). Forest Systems 19(1):36-50.

Wilhelmsen M. 2009. Coordinated investigation of living conditions 2008. Report No. 2009/40. Olso, Norway. Statistics Norway 2008:38-39.

Zandersen M, RSJ Tol. 2009. A meta-analysis of forest recreation values in Europe. Journal of Forest Economics 15:109-130.

Recibido: 03.07.15

Aceptado: 15.10.15 